\title{
Redshift Distortions as a Probe of Gravity
}

\author{
Eric V. Linder \\ Berkeley Lab, University of California, Berkeley, CA 94720, USA
}

(Dated: October 26, 2018)

\begin{abstract}
Redshift distortion measurements from galaxy surveys include sensitivity to the gravitational growth index distinguishing other theories from Einstein gravity. This gravitational sensitivity is substantially free from uncertainty in the effective equation of state of the cosmic expansion history. We also illustrate the bias in the traditional application to matter density determination using $f=\Omega_{m}(a)^{0.6}$, and how to avoid it.
\end{abstract}

\section{INTRODUCTION}

Measurements of growth of cosmic structure open windows on probing the theory of gravity and, in combination with observations of the expansion history, can distinguish between a physical dark energy and extended gravity as an explanation for the accelerating expansion of the universe. As pointed out by [1], the pattern of redshift distortions around large scale structure over cosmic time could offer such a probe of growth history. Recent developments such as the new VVDS redshift survey [1] and analysis of existing data sets [2] motivate examination of this prospective probe. This technique has the advantage of using galaxies purely as markers, ameliorating selection effects, and requiring only moderate resolution spectroscopy [1].

Redshift distortions are caused by velocity flows induced by gradients of the gravitational potential, with the gravitational potential evolving due to both the growth under gravitational attraction (given by general relativity or some other theory of gravity to be considered) and the dilution of the potentials due to the cosmic expansion (characterized by the effective equation of state). Because the velocity field involves one less spatial derivative of the potential than the density field does, the signal persists to larger, more linear scales, and offers the hope of serving as a cosmological and gravitational probe.

We investigate the sensitivity of this probe in $₫ \mathrm{II}$, calculating its leverage on distinguishing the theory of gravity and the equation of state. Its complementarity with expansion probes is considered in $₫$ III. In $₫$ IV we demonstrate how the traditional use of redshift distortions to determine the matter density becomes biased if the incorrect form is assumed.

\section{SENSITIVITY}

The redshift distortion parameter observed through the anisotropic pattern of galaxy redshifts on cluster scales is 3 ]

$$
\beta=\frac{1}{b} \frac{d \ln D}{d \ln a}=f / b,
$$

where $b$ is the bias between galaxies and the total matter, and $D$ is the linear theory growth factor at expansion scale factor $a$. Considering the gravitational growth index formalism of [4],

$$
f=\Omega_{m}(a)^{\gamma}
$$

where $\Omega_{m}(a)$ is the matter density as a fraction of the total energy density at scale factor $a$ and $\gamma$ is the gravitational growth index. Note that, as it was designed for, the growth index formalism separates out the two physical effects on the growth of structure and the redshift distortion: $\Omega_{m}(a)$ involves the expansion history and $\gamma$ focuses on the gravity theory. We will see that this is particularly beneficial in the present case.

To investigate the leverage of redshift distortion measurements at various redshifts on the cosmological parameters, we use a Fisher information matrix calculation to propagate uncertainties in $\beta$ to the estimations of the present matter density $\Omega_{m}$, the gravitational growth in$\operatorname{dex} \gamma$, and the effective equation of state parametrized by $w(a)=w_{0}+w_{a}(1-a)$, with $w_{0}$ the present effective dark energy equation of state, or pressure to energy density, ratio and $w_{a}$ a measure of its time variation. Initially we consider the bias parameter $b(a)$ to be well known. Figure 1 shows as a function of redshift the Fisher partial derivatives giving the sensitivities.

The gravitational growth index has substantial impact on the redshift distortion, with $\beta$ more sensitive to the growth index $\gamma$ than to the equation of state variables $w_{0}, w_{a}$, especially at low redshifts. This is furthermore where measurements can be made most precisely, so this suggests that redshift distortions offer a promising tool for investigating gravity. Sensitivity to the equation of state parameters is modest, so redshift distortions do not serve as a strong dark energy expansion probe. However, this insensitivity combined with the very different shapes of the $\gamma$ and $w_{0}, w_{a}$ curves mean that there is not much degeneracy between the gravity and the expansion history parameters - a promising sign.

Suppose we consider an optimistic scenario of $10 \%$ measurements of $\beta$ in 20 redshift bins from $z=0.1-2$. Note that current state of the art is a $37 \%$ measurement of $\beta(z=0.77)$ [1], $12 \%$ measurement (using additional clustering information) of $\beta(z=0.55)$ [2], and $18 \%$ measurement of $\beta(z=0.15)$ [5]. Additionally we include $\mathrm{CMB}$ constraints of $0.7 \%$ in the reduced distance 


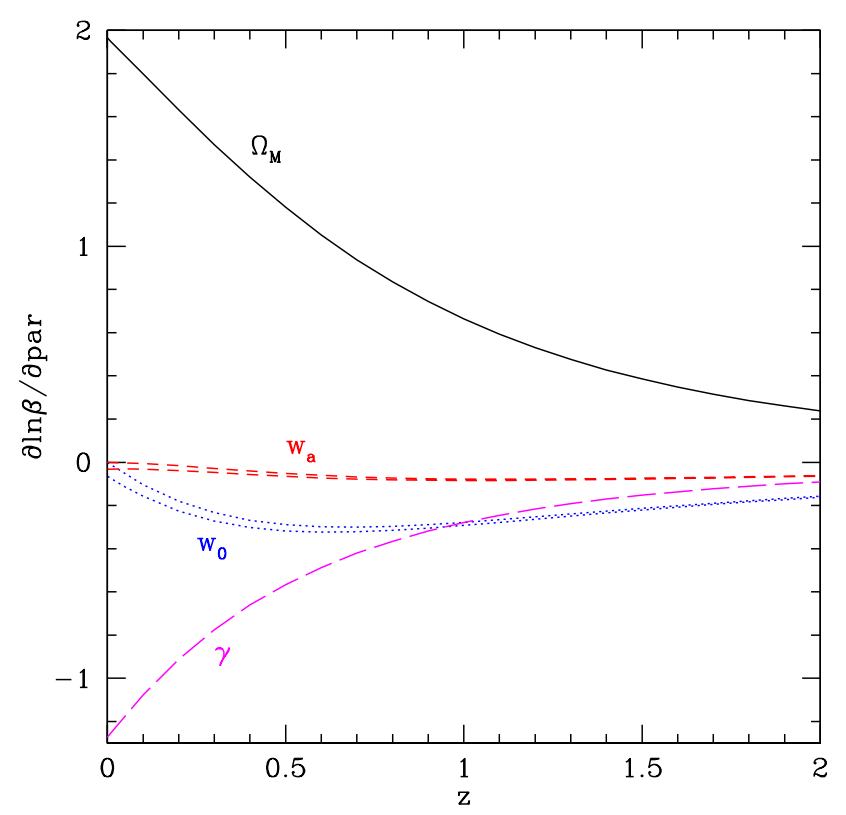

FIG. 1: The sensitivities of the redshift distortion $\beta$ to variations in the cosmological parameters. The unmarginalized uncertainty in a parameter is given by $\sigma(p)=\sigma_{\ln \beta} /|\partial \ln \beta / \partial p|$. For example, for a $10 \%$ precision on $\beta$ at $z=0.8$, the uncertainty on $w_{0}$ would be $0.1 / 0.3 \approx 0.3$. The upper curves for $w_{0}$ and $w_{a}$ show the sensitivity when the slight dependence of $\gamma$ on $w(z)$ is neglected.

to last scattering, à la Planck, which are independent of $\gamma$. The unmarginalized uncertainty in the determination of the gravitational growth index is $\sigma(\gamma)=0.048$. (Note that the difference between general relativity and the extradimensional DGP braneworld gravity scenario is $\Delta \gamma=0.13$.) If we marginalize over $\Omega_{m}$, keeping to the $\Lambda$ CDM $w=-1$ scenario, then $\sigma(\gamma)=0.061$, and if we also fit for a constant $w$ then this becomes 0.067 , and finally if we marginalize over a time varying $w(z)$ as well, i.e. $w_{0}$ and $w_{a}$, then the uncertainty is 0.077 .

The increase of $61 \%$ from unmarginalized to fully marginalized estimation is remarkably small. (By contrast, uncertainty in $w_{0}$ would increase by a factor 12 .) This reflects that $\gamma$ predominantly tests the gravity theory independent of the expansion history. (Indeed, $\delta \gamma \approx$ $\delta w / 20$.) The growth index estimation is also quite distinct from uncertainties in the matter density, with the correlation coefficient between $\gamma$ and $\Omega_{m}$ only 0.12 .

While from Fig. 1 we see that the main leverage on $\gamma$ arises from low redshift, the higher redshift measurements help not only through more statistics but in breaking the residual degeneracies. Thus, to achieve these constraints on $\gamma$ one must succeed in precision measurements of redshift distortions to $z>1$. If the data only goes out to $z=1$ then the marginalized uncertainty on $\gamma$ increases to 0.16 , while preserving the original statisti- cal power (e.g. by improving the precision by $\sqrt{2}$ of the now half as many measurements) yields an uncertainty of 0.12 , compared to the original ( $z=0.1-2$ data) value of 0.077 .

\section{COMPLEMENTARITY}

However, if we employ the redshift distortion method in complementarity with an expansion history probe such as Type Ia supernovae (SN), then we can obtain tight constraints on the gravitational growth index $\gamma$ with a more modest dataset in $\beta$, extending only over $z<1$. (Note that using baryon acoustic oscillations as the distance probe could be somewhat problematic, as they employ the same density field as the redshift distortions.) Combining redshift distortion measurements from $z=0.1-1$, plus CMB data, with SN observations from $z=0.1-1.7$ as from the future SNAP satellite (recall SN are purely an expansion probe, with no leverage on $\gamma$ ), we can fit for the growth index to 0.057 with negligible change in the SN determination of $w_{0}, w_{a}$. Thus the two methods work in parallel to test both gravity and dark energy.

Figure 2 shows the constraints in the $\Omega_{m}-\gamma$ plane, marginalizing over the equation of state parameters, with the combination of redshift distortion $\beta, \mathrm{CMB}$, and $\mathrm{SN}$ able to distinguish deviations in gravitational index.

Adding new parameters to describe the galaxy bias makes the situation more challenging. Because of the constraints from the distance data, the bias acts primarily to increase the uncertainty on $\gamma$. Considering two new parameters to describe the bias, $b(a)=b_{0}+b_{1}(1-a)$, one requires priors on these parameters of 0.03 (0.05) to prevent the estimation of $\gamma$ from increasing by more than $20 \%$ (40\%). For future surveys one can hope to limit the galaxy bias uncertainty through use of the higher order correlation function data present in the survey, and simulations taking advantage of that the velocity field probes larger, more linear scales than the density field.

Moreover, note that an additional low redshift $(z \approx$ $0.05)$ measurement helps considerably because there the growth factor involves nearly the present matter density (well determined by complementarity with the distance information), and the growth index, thus reducing the uncertainty on $\gamma$. The very optimistic situation of $5 \%$ measurements of redshift distortions from $z=0.1-1$, including at $z=0.05$ and priors of $0.03(0.05)$ on the bias parameters, with distance data, could allow a seven parameter fit, delivering knowledge of $\gamma$ to 0.041 (0.047).

\section{MATTER DENSITY (MIS)ESTIMATION}

Traditionally, redshift distortion measurements have been used to determine the matter density (see [5, 6, 6] and references therein). As seen in Fig. 1, $\beta$ has considerable sensitivity to $\Omega_{m}$. However, the correct form 


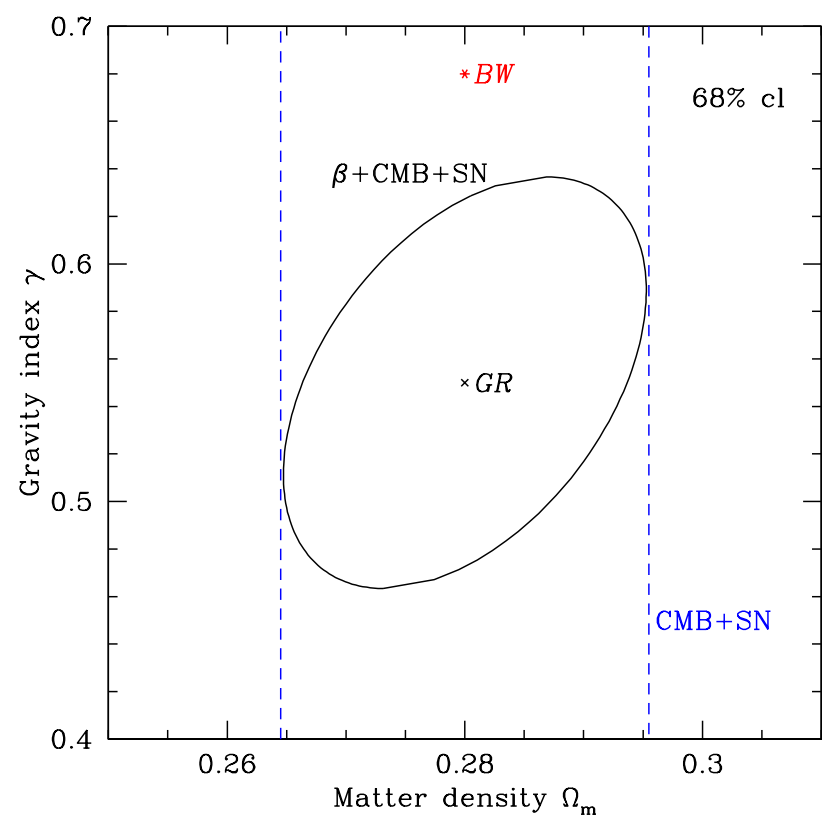

FIG. 2: Complementarity of measurements of the redshift distortion factor $\beta$, here ten data points from $z=0.1-1$ at $10 \%$ precision, with $\mathrm{CMB}$ and supernovae measurements of the expansion history, provides an appreciable test of gravity through the growth index $\gamma$. While the expansion probes are blind to $\gamma$ (vertical band), use of redshift distortion data can distinguish the Einstein value $\gamma=0.55$ (shown by the black $\mathrm{x}$ ) from braneworld gravity having $\gamma=0.68$ (red star).

for growth factor must be employed; the approximation $f=\Omega_{m}(a)^{0.6}$ from $1980[8]$ is appropriate only for a universe with purely matter, of which a fraction $\Omega_{m}$ clusters. A highly accurate growth index form is $f=\Omega_{m}(a)^{\gamma}$, with $\gamma=0.55+0.05[1+w(z=1)]$, [4] or simply $f=\Omega_{m}(a)^{0.55}$ for a $\Lambda \mathrm{CDM}$ universe. (And as shown in Fig. 1, ignoring the $w$ dependence of $\gamma$ is a good approximation.)

Figure 3illustrates the bias induced in the derived matter density if the incorrect form for the growth factor is employed. We see that using $f=\Omega_{m}(a)^{0.55}$ is at least 10 times more accurate than $f=\Omega_{m}(a)^{0.6}$. (The form of [9] improves on the 0.6 form by not quite a factor two; similar results on relative accuracy of the forms hold for dark energy other than $\Lambda$.) Using $f=\Omega_{m}(a)^{0.6}$ can bias the value of $\Omega_{m}$ derived from the measurements by $\sim 0.03$, a substantial offset for precision cosmology. By contrast, $f=\Omega_{m}(a)^{0.55}$ recovers $\Omega_{m}$ to better than 0.003 , with zero error relative to the exact numerical solution for growth in the important $z \approx 0.5$ range.

\section{CONCLUSION}

Testing gravity is an important experimental goal at the intersection of astrophysics and fundamental physics.

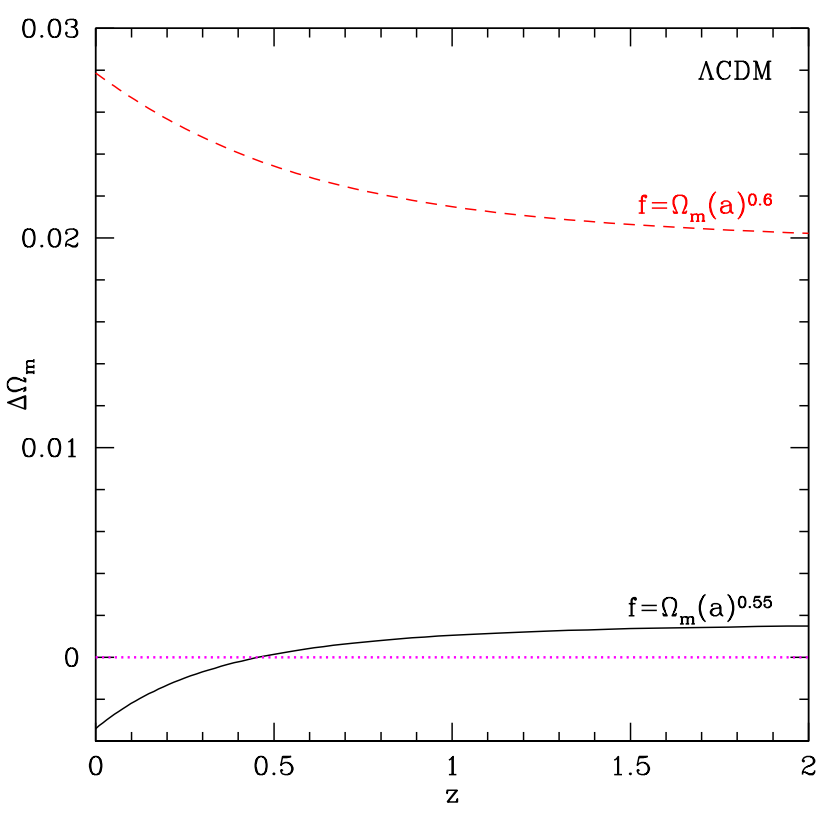

FIG. 3: Using redshift distortions to measure the matter density requires the proper form for the growth factor. The 1980 form (before CDM and dark energy) with growth index of 0.6 will misestimate $\Omega_{m}$ by up to 0.03 , while the modern form with 0.55 is more than 10 times as accurate.

The development of redshift distortion measurements in galaxy redshift surveys is an exciting advance. We have shown that $10 \%$ measurements from $z=0-1$, well within future observational capabilities, in complementarity with independent, accurate distances from $z=$ $0-1.7$ to measure the expansion history, can put significant limits on gravitational modifications. The growth and expansion methods work in parallel to test both gravity and dark energy.

Redshift distortions have little leverage on the dark energy equation of state but can determine the matter density accurately if the modern form $f=\Omega_{m}(a)^{0.55}$ is used.

While issues of galaxy bias and the exact relation between the velocity and matter power spectra (e.g. [3, 10]) add some uncertainty, further observational leverage exists also. According to [1], improved knowledge of the galaxy spatial correlation function could reduce error bars a factor of three, while larger surveys, such as extending the Vimos VLT Deep Survey (VVDS) to 400 $\operatorname{deg}^{2}$, could estimate $\beta$ to better than $5 \%$.

Redshift distortions are exciting especially because of the strong motivation for testing gravity on these length scales. While cosmological observations probe the theory of gravity on $1000 \mathrm{Mpc}$ scales, and galaxy dynamics on 1-100 kpc scales, velocity flows give insight on 1-30 Mpc scales where many theories predict a transition from the form causing the cosmic acceleration on large scales to 
the general relativity limit on small scales. As pointed out by [11], the relation between the velocity field of matter and the growth of density perturbations also probes the inverse square law of gravity, while [12] has emphasized that the redshift distortions have a different dependence on the metric potentials than do gravitational lensing shape distortions. Measuring redshift distortions, and in particular a value of $\gamma$ different from the Einstein value of 0.55 , could thus point the way to a new understanding of gravitation.

\section{Acknowledgments}

I thank Gigi Guzzo for motivating this investigation and the Aspen Center for Physics for providing the venue for such discussions. This work has been supported in part by the Director, Office of Science, Department of Energy under grant DE-AC02-05CH11231.

[8] P.J.E. Peebles, Large-Scale Structure of the Universe (Princeton U. Press, 1980)

[9] O. Lahav, M.J. Rees, P.B. Lilje, J.R. Primack, MNRAS 251, 128 (1991)

[10] R. Scoccimarro, Phys. Rev. D 70, 083007 (2004)

[11] P.J.E. Peebles, arXiv:astro-ph/0208037

[12] P. Zhang, M. Liguori, R. Bean, S. Dodelson, arXiv:0704.1932

[7] T. Matsubara \& Y. Suto, ApJL 470, L1 (1996) 